\title{
Resumen de tesis doctoral: Repercusión de la formación en adultos mayores: EI caso del Aula Abierta de Mayores ${ }^{13}$
}

Impact of training in the elderly: the case of the Open Classroom for the Elderly (doctoral thesis)

José Luis Rodríguez Díez

jlrdiez@gmail.com

Directores: Pérez de Guzmán Puya, M. V., Amador Muñoz, L. V., \& Moreno Crespo, P. A.

\section{Resumen}

La Encuesta Mayores (2010) establecía que en España un 60\% de las personas inician nuevas actividades tras la jubilación. Una de las demandas de esta nueva generación de adultos mayores es una formación universitaria de calidad. Esta investigación se plantea estudiar la repercusión de la formación en el adulto mayor en un contexto educativo formal, teniendo en cuenta las dimensiones social y psicológica. El trabajo está estructurado en dos partes: fundamentación teórica y marco empírico.

\section{Fundamentación teórica}

Esta parte está organizada en cuatro capítulos. En el primero, denominado "Aproximación al concepto de vejez", se presenta una revisión de las definiciones del término vejez, así como el origen de los distintos términos con los que se designa a esta etapa evolutiva y sus implicaciones. Se aborda una aproximación a la vejez a través de la historia, principalmente en Europa, y también la visión actual y futuro de la misma. Se realiza una revisión de legislación y documentos relevantes tanto en el ámbito internacional como el de la Comunidad Autónoma Andaluza (España) en esta etapa vital. Para explicar el envejecimiento se presentan diversos modelos teóricos, dedicando

\footnotetext{
${ }^{13}$ Recibido: 20/07/2015 Evaluado:04/09/2015 Aceptado: 10/09/2015
} 
un apartado a la jubilación, hecho que supone un punto de inflexión en la vida de las personas y para los mayores de 65 años la entrada oficial en la vejez.

El capítulo segundo, "La vejez como objeto de estudio", contempla un estudio evolutivo de la investigación sobre la vejez y el envejecimiento, sintetizando los trabajos llevados a cabo en geriatría, gerontología y la psicogerontología. Se profundizan las dimensiones psicológica y social, incorporando los aspectos familiares y de participación de los adultos mayores, por ser estas las dimensiones objeto de estudio de la investigación.

"El aprendizaje a lo largo de la vida" es el tercer capítulo, que presenta una reflexión sobre el aprendizaje a lo largo de la vida y su evolución desde la alfabetización de adultos. Se describen de forma pormenorizada los enfoques teóricos sobre el aprendizaje en los adultos. La simbiosis entre calidad de vida y envejecimiento activo está presente en este capítulo, como también el abordaje de la gerontagogía, como ámbito científico y profesional dedicado a la intervención socio-educativa con y para personas mayores.

El cuarto capítulo del marco teórico, titulado "Programas formativos para adultos mayores", analiza con profundidad los distintos programas para adultos mayores, con sus respectivas características. Se detiene en los programas universitarios para mayores, mediante una descripción de los modelos formativos. Por último, realiza un análisis en profundidad del programa "Aula Abierta de Mayores" de la Universidad Pablo de Olavide, por ser en este donde se ha llevado a cabo nuestra investigación. Se concluye con la legislación española más relevante sobre educación de adultos.

\section{Parte empírica}

La investigación se centra en analizar la influencia de la formación que reciben los estudiantes del Aula Abierta de Mayores de la Universidad Pablo de Olavide. Los objetivos giran en torno a la dimensión psicológica y social, destacando en la primera los aspectos personales y, en la segunda, los aspectos familiares y de participación. A partir de estas dimensiones se diseñan quince objetivos específicos. El objetivo general referido al aspecto personal pretende constatar la autoimagen y auto percepción que poseen los adultos. El objetivo, centrado en el aspecto familiar, trata de determinar la 
importancia de los mayores como soporte familiar, y referido al aspecto social pretende analizar la participación de los adultos mayores en el Aula Abierta de Mayores. La metodología utilizada ha sido de carácter mixto. Desde la perspectiva cuantitativa, el método seleccionado ha sido el no experimental, ex-post-facto o de caso único y para la elección de la muestra se ha utilizado un método no probabilístico casual.

\section{Conclusiones}

Los resultados han demostrado, a nivel general, cómo la asistencia al programa formativo supone:

En lo personal, mejora su autoestima, su autopercepción y el sentimiento de pertenencia al grupo de iguales y disminuye la sensación de rechazo. Asimismo, consideran que han cambiado cualitativamente respecto de los mayores de la generación anterior.

En lo familiar, han mejorado sus relaciones, proporcionándoles más objetividad a la hora de resolver problemas y permitiéndoles descubrir que la formación a lo largo de la vida tiene tanta importancia como sus tareas en el ámbito familiar.

En lo social, se sienten orgullosos de pertenecer al Aula Abierta y a la Universidad, aumentando sus expectativas con respecto a la formación a lo largo de la vida y generando la necesidad de seguir formándose cuando termine su participación en el programa formativo. 